\title{
On $q$-Analogues of the Barnes Multiple Zeta Functions
}

\author{
Yoshinori YAMASAKI
}

Kyushu University

(Communicated by H. Midorikawa)

\begin{abstract}
In this paper, we introduce $q$-analogues of the Barnes multiple zeta functions. We show that these functions can be extended meromorphically to the whole plane, and moreover, tend to the Barnes multiple zeta functions when $q \uparrow 1$ for all complex numbers.
\end{abstract}

\section{Introduction}

The aim of the present paper is to introduce $q$-analogues of the Barnes multiple zeta function ([3]);

$$
\zeta_{r}(s, z ; \omega):=\sum_{n_{1}, \ldots, n_{r} \geq 0}\left(n_{1} \omega_{1}+\cdots+n_{r} \omega_{r}+z\right)^{-s} \quad(\operatorname{Re}(s)>r)
$$

where $\omega_{1}, \ldots, \omega_{r}$ are complex parameters which lie on some half plane. We study an analytic continuation of the $q$-analogue of $\zeta_{r}(s, z ; \omega)$. We determine especially, true $q$-analogues of the Barnes multiple zeta function when $\omega_{i}=1(1 \leq i \leq r)$. Here, by a true $q$-analogue, we mean when the classical limit $q \uparrow 1$ of the $q$-analogue reproduces the original zeta function for all $s \in \mathbf{C}$. Recall the Hurwitz zeta function's case, that is, the case $r=1$. Let $0<q<1$ and $[z]_{q}:=\left(1-q^{z}\right) /(1-q)$ for $z \in \mathbf{C}$. In [6] (see also [5]) we studied $q$-analogues of the Hurwitz zeta function $\zeta(s, z):=\sum_{n=0}^{\infty}(n+z)^{-s}$ defined via the $q$-series with two complex variables $s, t \in \mathbf{C}$;

$$
\tilde{\zeta}_{q}(s, t, z):=\sum_{n=0}^{\infty} \frac{q^{(n+z) t}}{[n+z]_{q}{ }^{s}} \quad(\operatorname{Re}(t)>0) .
$$

The function $\tilde{\zeta}_{q}(s, t, z)$ is continued meromorphically to the whole $s, t$-plane. We obtained the necessary and sufficient condition for the variable $t \in \mathbf{C}$ so that $\tilde{\zeta}_{q}(s, t, z)$ is a true $q$-analogue of $\zeta(s, z)$. Namely, these functions $\tilde{\zeta}_{q}^{(v)}(s, z):=\tilde{\zeta}_{q}(s, s-v, z)(v \in \mathbf{N})$ give true $q$-analogues Received February 8, 2005 2000 Mathematics Subject Classification: Primary 11M41, 11M35

Key words and phrases: Barnes' multiple zeta functions, Hurwitz's zeta functions, $q$-series, $q$-binomial coefficients, classical limit. 
of the Hurwitz zeta function among the functions of the form $\tilde{\zeta}_{q}(s, \varphi(s), z)$ where $\varphi(s)$ is a meromorphic function on $\mathbf{C}$. The main purpose is to generalize the results in [6] to $r \geq 1$.

The plan of this paper is as follows. In Section 2, we define a $q$-analogue $\zeta_{q, r}(s, t, z)$ of the Barnes multiple zeta function for $\omega_{i}=1(1 \leq i \leq r)$ and give the main theorem (Theorem 2.1). In Section 3, we first study an analytic continuation of the $q$-analogue $\zeta_{q, r}(s, t, z)$ and then prove the main theorem. In Section 4 , we study a $q$-analogue $\zeta_{q, r}(s, t, z ; \omega)$ of the multiple zeta functions for general parameters $\boldsymbol{\omega}$. Using the binomial theorem, we give an analytic continuation of the $q$-analogue (Proposition 4.1). In the appendix, we introduce a $q$-analogue $\tilde{\Gamma}_{q}(z)$ of the gamma function $\Gamma(z)$ associated to the $q$ analogue $\tilde{\zeta}_{q}(s, t, z)$ of the Hurwitz zeta function. We first observe fundamental properties of $\tilde{\Gamma}_{q}(z)$. The rest of the appendix is devoted to study $q$-analogues of the limit formula of Lerch (Proposition A.3) and the Gauss-Legendre formula (Proposition A.5).

Throughout the paper, we assume $0<q<1$. We put $[n]_{q} !:=[n]_{q}[n-1]_{q} \cdots[1]_{q}$ for $n \in \mathbf{N}$. Further, for non-negative integers $m$ and $n$, we define the $q$-binomial coefficient $\left[\begin{array}{c}m \\ n\end{array}\right]_{q}$ by

$$
\left[\begin{array}{l}
m \\
n
\end{array}\right]_{q}:=\frac{(q ; q)_{m}}{(q ; q)_{n}(q ; q)_{m-n}}
$$

where $(a ; q)_{m}:=\prod_{l=0}^{m-1}\left(1-a q^{l}\right)$ for $m \geq 1$ and $(a ; q)_{0}:=1$. We denote the field of complex numbers, the ring of rational integers and the set of positive integers by $\mathbf{C}, \mathbf{Z}$ and $\mathbf{N}$ respectively. Also, if $Q$ is a set, $Q_{P}$ stands for the set of all elements in $Q$ which satisfy the condition $P$.

\section{Definition of $q$-analogues and the main theorem}

Let $s, t \in \mathbf{C}$ and $z \notin-\mathbf{Z}_{\leq 0}$. We study a $q$-analogue of the Barnes multiple zeta function

$$
\zeta_{r}(s, z):=\sum_{n_{1}, \ldots, n_{r} \geq 0}\left(n_{1}+\cdots+n_{r}+z\right)^{-s}
$$

defined by the following $q$-series;

$$
\zeta_{q, r}(s, t, z):=\sum_{n_{1}, \ldots, n_{r} \geq 0} \frac{q^{n_{1} t+n_{2}(t-1)+\cdots+n_{r}(t-r+1)}}{\left[n_{1}+\cdots+n_{r}+z\right]_{q}^{s}} .
$$

The series $\zeta_{q, r}(s, t, z)$ converges absolutely for $\operatorname{Re}(t)>r-1$. When $r=1$, we put $\zeta_{q}(s, t, z):=\zeta_{q, 1}(s, t, z)$. In view of the results in [6], we put $\zeta_{q, r}^{(v)}(s, z):=\zeta_{q, r}(s, s-v, z)$ and $\zeta_{q}^{(v)}(s, z):=\zeta_{q}(s, s-v, z)$ for $v \in \mathbf{N}$. The following theorem is the main result of this paper. 
THEOREM 2.1. Let $t=\varphi(s)$ be a meromorphic function on $\mathbf{C}$. Then the formula

$$
\lim _{q \uparrow 1} \zeta_{q, r}(s, \varphi(s), z)=\zeta_{r}(s, z) \quad(s \in \mathbf{C})
$$

holds if and only if the function $\varphi(s)$ can be written as $\varphi(s)=s-v$ for some $v \in \mathbf{N}$.

REMARK 2.2. (i) By Theorem 2.1, it is clear that the functions of the type $\sum_{v \text { :finite }} a_{q}^{(v)}(s, z) \zeta_{q, r}^{(v)}(s, z)$ for some holomorphic functions $a_{q}^{(v)}(s, z)$ satisfying $\lim _{q \uparrow 1} \sum_{v \text { : finite }} a_{q}^{(v)}(s, z)=1$ are also true $q$-analogues of $\zeta_{r}(s, z)$. Note that the $q$-analogue of the Hurwitz zeta function discussed in [6] is given by $\tilde{\zeta}_{q}^{(v)}(s, z)=\zeta_{q}^{(v)}(s, z) \times q^{z(s-v)}$.

(ii) The $q$-analogue of the Hurwitz zeta function studied in [10] is different from ours. It is not of the form of the $(q-)$ Dirichlet series and, in fact, is needed an extra term (precisely, see [6, Corollary 2.4]).

It is easy to see that $\zeta_{r}(s, z)$ is expressed as

$$
\zeta_{r}(s, z)=\sum_{n=0}^{\infty}\left(\begin{array}{c}
n+r-1 \\
r-1
\end{array}\right)(n+z)^{-s} .
$$

To obtain a similar expression for $\zeta_{q, r}(s, t, z)$, we need the following lemma.

LEMMA 2.3. (i) For $l, m \in \mathbf{Z}_{\geq 0}$, it holds that

$$
\sum_{d=0}^{l}\left[\begin{array}{c}
m-1+d \\
m-1
\end{array}\right]_{q} q^{d}=\left[\begin{array}{c}
m+l \\
m
\end{array}\right]_{q} .
$$

(ii) For $r \in \mathbf{N}$, it holds that

$$
\sum_{\substack{n_{1}, \ldots, n_{r} \geq 0 \\
n_{1}+\cdots+n_{r}=n}} q^{n_{1}+2 n_{2}+\cdots+r n_{r}}=q^{n}\left[\begin{array}{c}
n+r-1 \\
r-1
\end{array}\right]_{q} .
$$

PROOF. The formula (2.2) is well-known (see [1], also [4]). We show the formula (2.3) by induction on $r$. It is clear that (2.3) holds for $r=1$. Suppose it holds for $r-1$. Then the left hand side of (2.3) is equal to

$$
\sum_{n_{1}=0}^{n} q^{n_{1}+\left(n-n_{1}\right)} \sum_{\substack{n_{2}, \ldots, n_{r} \geq 0 \\
n_{2}+\cdots+n_{r}=n-n_{1}}} q^{n_{2}+2 n_{3}+\cdots+(r-1) n_{r}}=q^{n} \sum_{n_{1}=0}^{n}\left[\begin{array}{c}
n_{1}+r-2 \\
r-2
\end{array}\right]_{q} q^{n_{1}}
$$

Using the formula (2.2) for $l=n, m=r-1$ and $d=n_{1}$, we obtain the desired formula.

PROPOSITION 2.4. It holds that

$$
\zeta_{q, r}(s, t, z)=\sum_{n=0}^{\infty}\left[\begin{array}{c}
n+r-1 \\
r-1
\end{array}\right]_{q} \frac{q^{n(t-r+1)}}{[n+z]_{q}{ }^{s}} .
$$


PROOF. It is easy to see that

$$
\begin{aligned}
\zeta_{q, r}(s, t, z) & =\sum_{n=0}^{\infty} \sum_{\substack{n_{1}, \ldots, n_{r} \geq 0 \\
n_{1}+\cdots+n_{r}=n}} \frac{q^{(t+1)\left(n_{1}+\cdots+n_{r}\right)-\left(n_{1}+2 n_{2}+\cdots+r n_{r}\right)}}{\left[n_{1}+\cdots+n_{r}+z\right]_{q}^{s}} \\
& =\sum_{n=0}^{\infty} \frac{q^{(t+1) n}}{[n+z]_{q}^{s}} \sum_{\substack{n_{1}, \ldots, n_{r} \geq 0 \\
n_{1}+\cdots+n_{r}=n}} q^{-\left(n_{1}+2 n_{2}+\cdots+r n_{r}\right)} .
\end{aligned}
$$

Substituting $q^{-1}$ for $q$ into (2.3) yields

$$
\sum_{\substack{n_{1}, \ldots, n_{r} \geq 0 \\
n_{1}+\cdots+n_{r}=n}} q^{-\left(n_{1}+2 n_{2}+\cdots+r n_{r}\right)}=q^{-n}\left[\begin{array}{c}
n+r-1 \\
r-1
\end{array}\right]_{q^{-1}}=q^{-n r}\left[\begin{array}{c}
n+r-1 \\
r-1
\end{array}\right]_{q} .
$$

Hence we obtain the formula (2.4).

\section{Proof of the main theorem}

In this section, we give a proof of Theorem 2.1. We first provide analytic continuations of $\zeta_{r}(s, z)$ with respect to $s$ (see [9]) and study of $\zeta_{q, r}(s, t, z)$ with respect to $t$. Since we have the following ladder relations

$$
\begin{aligned}
\zeta_{r}(s, z) & =\zeta_{r}(s, z+1)+\zeta_{r-1}(s, z), \\
\zeta_{q, r}(s, t, z) & =q^{t-r+1} \zeta_{q, r}(s, t, z+1)+\zeta_{q, r-1}(s, t, z),
\end{aligned}
$$

it is sufficient to study the analytic continuation when $\operatorname{Re}(z)>0$. Here we understand $\zeta_{0}(s, z)=z^{-s}$ and $\zeta_{q, 0}(s, t, z)=[z]_{q}{ }^{-s}$.

3.1. An analytic continuation of $\zeta_{r}(s, z)$. For each $l \in \mathbf{Z}_{\geq 0}$, we put $(x)_{l}:=x(x+$ 1) $\cdots(x+l-1)=\Gamma(x+l) / \Gamma(x)$. Then $(x)_{l}$ can be written as $(x)_{l}=\sum_{j=0}^{l} s(l, j) x^{j}$ where $s(l, j)$ is the Stirling number of the first kind. Hence we have

$$
\left(\begin{array}{c}
n+r-1 \\
r-1
\end{array}\right)=\frac{(n)_{r}}{n(r-1) !}=\frac{1}{(r-1) !} \sum_{j=0}^{r} s(l, j) n^{j-1}=\sum_{l=0}^{r-1} P_{r}^{l}(z)(n+z)^{l},
$$

where $P_{r}^{l}(z)(0 \leq l \leq r-1)$ is a polynomial in $z$ defined by

$$
P_{r}^{l}(z):=\frac{1}{(r-1) !} \sum_{j=l}^{r-1}\left(\begin{array}{l}
j \\
l
\end{array}\right) s(r, j+1)(-z)^{j-l} .
$$

Thus, we have by (2.1)

$$
\zeta_{r}(s, z)=\sum_{l=0}^{r-1} P_{r}^{l}(z) \zeta(s-l, z)
$$


Recall also the Euler-Maclaurin summation formula (see, e.g., [1, p. 619]): For $a, b \in \mathbf{Z}$ satisfying $a<b$, a $C^{\infty}$-function $f(x)$ on $[a, \infty)$, and an arbitrary integer $M \geq 0$, we have

$$
\begin{aligned}
\sum_{n=a}^{b} f(n)= & \int_{a}^{b} f(x) d x+\frac{1}{2}(f(a)+f(b)) \\
& +\sum_{k=1}^{M} \frac{B_{k+1}}{(k+1) !}\left(f^{(k)}(b)-f^{(k)}(a)\right) \\
& -\frac{(-1)^{M+1}}{(M+1) !} \int_{a}^{b} \tilde{B}_{M+1}(x) f^{(M+1)}(x) d x,
\end{aligned}
$$

where $B_{k}$ is the Bernoulli number and $\tilde{B}_{k}(x)$ is the periodic Bernoulli polynomial defined by $\tilde{B}_{k}(x)=B_{k}(x-\lfloor x\rfloor)$ with $\lfloor x\rfloor$ being the largest integer not exceeding $x$. Putting $f(x):=$ $(x+z)^{-s}$, we obtain

$$
\begin{aligned}
\zeta(s, z)= & \frac{1}{s-1} z^{-s+1}+\frac{1}{2} z^{-s}+\sum_{k=1}^{M} \frac{B_{k+1}}{(k+1) !}(s)_{k} z^{-s-k} \\
& -\frac{(s)_{M+1}}{(M+1) !} \int_{0}^{\infty} \tilde{B}_{M+1}(x)(x+z)^{-s-M-1} d x .
\end{aligned}
$$

Since $\operatorname{Re}(z)>0$, the equation (3.4) gives an analytic continuation of the Hurwitz zeta function $\zeta(s, z)$ to the region $\operatorname{Re}(s)>-M$. Therefore, by (3.2) and (3.4), we obtain the following

PROPOSITION 3.1. For any integers $M_{l} \geq 0(0 \leq l \leq r-1)$, we have

$$
\begin{aligned}
\zeta_{r}(s, z)= & \sum_{l=0}^{r-1} \frac{P_{r}^{l}(z)}{s-l-1} z^{-s+l+1}+\frac{1}{2} \sum_{l=0}^{r-1} P_{r}^{l}(z) z^{-s+l} \\
& +\sum_{l=0}^{r-1} P_{r}^{l}(z) \sum_{k_{l}=1}^{M_{l}} \frac{B_{k_{l}+1}}{\left(k_{l}+1\right) !}(s-l)_{k_{l}} z^{-s+l-k_{l}} \\
& -\sum_{l=0}^{r-1} \frac{P_{r}^{l}(z)(s-l)_{M_{l}+1}}{\left(M_{l}+1\right) !} \int_{0}^{\infty} \tilde{B}_{M_{l}+1}(x)(x+z)^{-s+l-M_{l}-1} d x .
\end{aligned}
$$

This gives an analytic continuation of $\zeta_{r}(s, z)$ to the region $\operatorname{Re}(s)>M$ where $M:=$ $\max \left\{-M_{l}+l \mid 0 \leq l \leq r-1\right\}$.

3.2. An analytic continuation of $\zeta_{q, r}(s, t, z)$. It is easy to see that

$$
\left[\begin{array}{c}
n+r-1 \\
r-1
\end{array}\right]_{q}=\frac{1}{[r-1]_{q} !} \prod_{j=1}^{r-1} \frac{1-q^{n+z}+q^{n+z}-q^{n+j}}{1-q}
$$




$$
=\frac{1}{[r-1]_{q} !} \prod_{j=1}^{r-1}\left([n+z]_{q}-q^{n+j}[z-j]_{q}\right)=\sum_{l=0}^{r-1} q^{n(r-1-l)} P_{q, r}^{l}(z)[n+z]_{q}^{l},
$$

where $P_{q, r}^{l}(z)(0 \leq l \leq r-1)$ is a function of $z$ defined by

$$
P_{q, r}^{l}(z):=\frac{(-1)^{r-1-l}}{[r-1]_{q} !} \sum_{1 \leq m_{1}<\cdots<m_{r-1-l} \leq r-1} q^{m_{1}+\cdots+m_{r-1-l}}\left[z-m_{1}\right]_{q} \cdots\left[z-m_{r-1-l}\right]_{q}
$$

for $0 \leq l \leq r-2$ and $P_{q, r}^{r-1}(z):=1 /[r-1]_{q} !$. Therefore we have by (2.4)

$$
\zeta_{q, r}(s, t, z)=\sum_{l=0}^{r-1} P_{q, r}^{l}(z) \zeta_{q}(s-l, t-l, z)
$$

For example, we have

$$
\begin{aligned}
\zeta_{q, 2}(s, t, z)= & \zeta_{q}(s-1, t-1, z)-q[z-1]_{q} \zeta_{q}(s, t, z), \\
\zeta_{q, 3}(s, t, z)= & \frac{1}{1+q}\left\{\zeta_{q}(s-2, t-2, z)\right. \\
& -\left(q[z-1]_{q}+q^{2}[z-2]_{q}\right) \zeta_{q}(s-1, t-1, z) \\
& \left.+q^{3}[z-1]_{q}[z-2]_{q} \zeta_{q}(s, t, z)\right\} .
\end{aligned}
$$

We now recall the analytic continuation of $\zeta_{q}(s, t, z)$ proved in [6]. Let $N \in \mathbf{N}$. Put $f_{q}(x):=$ $q^{x t}\left(1-q^{x+z}\right)^{-s}$. Define the polynomial $b_{j}^{\varepsilon}(s)(0 \leq \varepsilon \leq j)$ in $s$ by the following equation:

$$
\frac{d^{j}}{d x^{j}}\left\{\left(1-q^{x+z}\right)^{-s}\right\}=(\log q)^{j} \sum_{\varepsilon=0}^{j} b_{j}^{\varepsilon}(s)\left(1-q^{x+z}\right)^{-s-\varepsilon} .
$$

By the Leibniz rule, we have

$$
f_{q}^{(k)}(x)=(\log q)^{k} q^{x t} \sum_{\varepsilon=0}^{k} c_{k}^{\varepsilon}(s, t)\left(1-q^{x+z}\right)^{-s-\varepsilon}, \quad c_{k}^{\varepsilon}(s, t):=\sum_{j=\varepsilon}^{k}\left(\begin{array}{l}
k \\
j
\end{array}\right) t^{k-j} b_{j}^{\varepsilon}(s) .
$$

Choosing $f(x)=f_{q}(x)$ and $M=N$ in (3.3), we have

$$
\begin{aligned}
\zeta_{q}(s, t, z)= & \frac{1}{2}\left(\frac{1-q^{z}}{1-q}\right)^{-s}-\sum_{k=1}^{N} \sum_{\varepsilon=0}^{k} \frac{B_{k+1}}{(k+1) !} c_{k}^{\varepsilon}(s, t)\left(\frac{1-q^{z}}{1-q}\right)^{-s-\varepsilon} \frac{(\log q)^{k}}{(1-q)^{\varepsilon}} \\
& +(1-q)^{s} I_{q, 0}^{0}(s, t, z)+\frac{(-1)^{N}(\log q)^{N+1}(1-q)^{s}}{(N+1) !} \\
& \times \sum_{\varepsilon=0}^{N+1} c_{N+1}^{\varepsilon}(s, t) I_{q, \varepsilon}^{N+1}(s, t, z),
\end{aligned}
$$


where

$$
I_{q, \varepsilon}^{m}(s, t, z):=\int_{0}^{\infty} \tilde{B}_{m}(x) q^{x t}\left(1-q^{x+z}\right)^{-s-\varepsilon} d x .
$$

Note that $\tilde{B}_{0}(x)=1$. Recall now the Fourier expansion of $\tilde{B}_{m}(x)$ (see, e.g., [11, p. 191]);

$$
\tilde{B}_{m}(x)=-m ! \sum_{n \in \mathbf{Z} \backslash\{0\}} \frac{e^{2 \pi \sqrt{-1} n x}}{(2 \pi \sqrt{-1} n)^{m}} \quad(m \geq 2) .
$$

Put $u=q^{x+z}$. Then we have

$$
\begin{aligned}
& I_{q, 0}^{0}(s, t, z)=-\frac{q^{-z t}}{\log q} b_{q^{z}}(t,-s+1), \\
& I_{q, \varepsilon}^{m}(s, t, z)=\sum_{n \in \mathbf{Z} \backslash\{0\}} \frac{m ! e^{-2 \pi \sqrt{-1}} n z}{(2 \pi \sqrt{-1} n)^{m}} \frac{q^{-z t}}{\log q} b_{q^{z}}(\delta n+t,-s-\varepsilon+1) \quad(m \geq 2),
\end{aligned}
$$

where $\delta=2 \pi \sqrt{-1} / \log q$. Here $b_{w}(\alpha, \beta)$ is the incomplete beta function defined by the integral

$$
b_{w}(\alpha, \beta):=\int_{0}^{w} u^{\alpha-1}(1-u)^{\beta-1} d u \quad(0<\operatorname{Re}(w)<1) .
$$

This integral converges absolutely for $\operatorname{Re}(\alpha)>0$. Hence the function $b_{w}(\alpha, \beta)$ is holomorphic for $\operatorname{Re}(\alpha)>0$ and for all $\beta \in \mathbf{C}$. Note that if $\operatorname{Re}(\beta)>0$, we have $\lim _{w \rightarrow 1} b_{w}(\alpha, \beta)=$ $\mathrm{B}(\alpha, \beta)$ where $\mathrm{B}(\alpha, \beta)$ is the beta function. Further, for any integer $N^{\prime} \geq 2$, repeated use of integration by parts yields

$$
\begin{aligned}
b_{w}(\alpha, \beta)= & \sum_{l=1}^{N^{\prime}-1}(-1)^{l-1} \frac{(1-\beta)_{l-1}}{(\alpha)_{l}} w^{\alpha+l-1}(1-w)^{\beta-l} \\
& +(-1)^{N^{\prime}-1} \frac{(1-\beta)_{N^{\prime}-1}}{(\alpha)_{N^{\prime}-1}} b_{w}\left(\alpha+N^{\prime}-1, \beta-N^{\prime}+1\right) .
\end{aligned}
$$

As a function of $\alpha$, this expression gives an analytic continuation of $b_{w}(\alpha, \beta)$ to the region $\operatorname{Re}(\alpha)>1-N^{\prime}$. Hence the functions $I_{q, 0}^{0}(s, t, z)$ and $I_{q, \varepsilon}^{m}(s, t, z)$ are meromorphically continued to the region $\operatorname{Re}(t)>1-N^{\prime}$ for any integer $N^{\prime} \geq 2$. Let $M \geq 0$ be an arbitrary large integer. Using the expressions (3.8) and (3.9), and applying the formula (3.10) to $I_{q, \varepsilon}^{m}(s, t, z)$ with $N^{\prime}:=M-N+1 \geq 2$, we see that the formula (3.6) can be written as

$$
\begin{aligned}
\zeta_{q}(s, t, z)= & -\frac{q^{-z t}(1-q)^{s}}{\log q} b_{q^{z}}(t,-s+1)+\frac{1}{2}\left(\frac{1-q^{z}}{1-q}\right)^{-s} \\
& +D_{q}^{1}(s, t, z ; N, M)+D_{q}^{2}(s, t, z ; N, M)+D_{q}^{3}(s, t, z ; N, M),
\end{aligned}
$$


where

$$
\begin{aligned}
D_{q}^{1}(s, t, z ; N, M):= & -\sum_{k=1}^{N} \sum_{\varepsilon=0}^{k} \frac{B_{k+1}}{(k+1) !} c_{k}^{\varepsilon}(s, t)\left(\frac{1-q^{z}}{1-q}\right)^{-s-\varepsilon} \frac{(\log q)^{k}}{(1-q)^{\varepsilon}}, \\
D_{q}^{2}(s, t, z ; N, M):= & \sum_{\varepsilon=0}^{N+1} \sum_{l=1}^{M-N} \sum_{n \in \mathbf{Z} \backslash\{0\}} \frac{(-1)^{N+l-1}}{(2 \pi \sqrt{-1} n)^{N+1}} \frac{c_{N+1}^{\varepsilon}(s, t)(s+\varepsilon)_{l-1} q^{z(l-1)}}{(1-q)^{l}(\delta n+t)_{l}} \\
& \times\left(\frac{1-q^{z}}{1-q}\right)^{-s-\varepsilon+1-l} \frac{(\log q)^{N}}{(1-q)^{\varepsilon-1}}, \\
D_{q}^{3}(s, t, z ; N, M):= & \sum_{\varepsilon=0}^{N+1} \sum_{n \in \mathbf{Z} \backslash\{0\}} \frac{(-1)^{M+1}}{(2 \pi \sqrt{-1} n)^{N+1}} \frac{c_{N+1}^{\varepsilon}(s, t)(s+\varepsilon)_{M-N} q^{z(M-N)}}{(1-q)^{M-N}(\delta n+t)_{M-N}} \\
& \times \frac{(\log q)^{N+1}}{(1-q)^{\varepsilon}} \int_{0}^{\infty} e^{2 \pi \sqrt{-1} n x} q^{x(t+M-N)}\left(\frac{1-q^{x+z}}{1-q}\right)^{-s-\varepsilon-M+N} d x .
\end{aligned}
$$

The equation (3.11) gives an analytic continuation of $\zeta_{q}(s, t, z)$ to the region $\operatorname{Re}(t)>1-$ $N^{\prime}=N-M$. Note that, by the fact $c_{k}^{k}(s, t)=(s)_{k}$ and (3.7) again, we have

$$
\begin{aligned}
& \lim _{q \uparrow 1} D_{q}^{1}(s, t, z ; N, M)=\sum_{k=1}^{N} \frac{B_{k+1}}{(k+1) !}(s)_{k} z^{-s-k}, \\
& \lim _{q \uparrow 1} D_{q}^{2}(s, t, z ; N, M)=\sum_{l=N+1}^{M} \frac{B_{l+1}}{(l+1) !}(s)_{l} z^{-s-l}, \\
& \lim _{q \uparrow 1} D_{q}^{3}(s, t, z ; N, M)=-\frac{(s)_{M+1}}{(M+1) !} \int_{0}^{\infty} \tilde{B}_{M+1}(x)(x+z)^{-s-M-1} d x .
\end{aligned}
$$

Therefore, by (3.5) and (3.11), we obtain the following

Proposition 3.2. For any integers $N_{l} \geq 1$ and $M_{l} \geq N_{l}+1(0 \leq l \leq r-1)$, we have

$$
\begin{aligned}
\zeta_{q, r}(s, t, z)= & -\frac{(1-q)^{s-(r-1)}}{\log q} \sum_{l=0}^{r-1} P_{q, r}^{l}(z) q^{-z(t-l)}(1-q)^{r-1-l} b_{q^{z}}(t-l,-s+l+1) \\
& +\frac{1}{2} \sum_{l=0}^{r-1} P_{q, r}^{l}(z)\left(\frac{1-q^{z}}{1-q}\right)^{-s+l}+\sum_{l=0}^{r-1} P_{q, r}^{l}(z) D_{q}^{1}\left(s-l, t-l, z ; N_{l}, M_{l}\right) \\
& +\sum_{l=0}^{r-1} P_{q, r}^{l}(z) D_{q}^{2}\left(s-l, t-l, z ; N_{l}, M_{l}\right) \\
& +\sum_{l=0}^{r-1} P_{q, r}^{l}(z) D_{q}^{3}\left(s-l, t-l, z ; N_{l}, M_{l}\right) .
\end{aligned}
$$


This gives an analytic continuation of $\zeta_{q, r}(s, t, z)$ to the region $\operatorname{Re}(t)>M^{\prime}$ where $M^{\prime}:=$ $\max \left\{N_{l}-M_{l}+l \mid 0 \leq l \leq r-1\right\}$.

3.3. Proof of Theorem 2.1. Note the following lemma.

LEMMA 3.3. It holds that

$$
\lim _{q \uparrow 1} P_{q, r}^{l}(z)=P_{r}^{l}(z)
$$

PROOF. By the definition of $P_{q, r}^{l}(z)$, it is sufficient to show

$$
\begin{gathered}
(-1)^{r-1-l} \sum_{1 \leq m_{1}<\cdots<m_{r-1-l} \leq r-1}\left(z-m_{1}\right) \cdots\left(z-m_{r-1-l}\right) \\
=\sum_{k=l}^{r-1}\left(\begin{array}{l}
j \\
l
\end{array}\right) s(r, j+1)(-z)^{j-l} .
\end{gathered}
$$

Notice that the left hand side of (3.15) is equal to the coefficient of $x^{l}$ in the polynomial $p_{r}(x):=\prod_{j=1}^{r-1}(x-(z-j))$ in $x$. Since $p_{r}(x)=(x-z)_{r} /(x-z)$, we have

$$
p_{r}(x)=\sum_{j=0}^{r-1} s(r, j+1)(x-z)^{j}=\sum_{l=0}^{r-1}\left(\sum_{j=l}^{r-1}\left(\begin{array}{l}
j \\
l
\end{array}\right) s(r, j+1)(-z)^{j-l}\right) x^{l} .
$$

Hence the desired formula follows.

We are ready to prove the main theorem.

Proof of TheOrem 2.1. We first show the sufficiency. Let $t=s-v$ for $v \in$ N. Notice that, by (3.5), we have $\zeta_{q, r}^{(v)}(s, z)=\sum_{l=0}^{r-1} P_{q, r}^{l}(z) \zeta_{q}^{(v)}(s-l, z)$. Hence, by [6, Theorem 2.1], Lemma 3.3 and (3.2), we have

$$
\lim _{q \uparrow 1} \zeta_{q, r}^{(v)}(s, z)=\sum_{l=0}^{r-1} P_{r}^{l}(z) \zeta(s-l, z)=\zeta_{r}(s, z) \quad(s \in \mathbf{C}) .
$$

We next show the necessity. Suppose that $\lim _{q \uparrow 1} \zeta_{q, r}(s, t, z)$ exists and satisfies $\lim _{q \uparrow 1} \zeta_{q, r}(s, t, z)=\zeta_{r}(s, z)$ for all $s \in \mathbf{C}$ with some meromorphic function $t=\varphi(s)$. Then, by Proposition 3.1, Proposition 3.2, Lemma 3.3, (3.12), (3.13) and (3.14), it is necessary to hold

$$
\begin{aligned}
-\lim _{q \uparrow 1} & \frac{(1-q)^{s-(r-1)}}{\log q} \sum_{l=0}^{r-1} P_{q, r}^{l}(z) q^{-z(t-l)}(1-q)^{r-1-l} b_{q^{z}}(t-l,-s+l+1) \\
= & \sum_{l=0}^{r-1} \frac{P_{r}^{l}(z)}{s-l-1} z^{-s+l+1} .
\end{aligned}
$$


Assume $\operatorname{Re}(s)<1$. Since $\lim _{q \uparrow 1}(1-q)^{s-(r-1)} / \log q$ diverges, it is necessary to hold

$$
\lim _{q \uparrow 1} \sum_{l=0}^{r-1} P_{q, r}^{l}(z) q^{-z(t-l)}(1-q)^{r-1-l} b_{q z}(t-l,-s+l+1)=0 .
$$

Notice that $\lim _{q \uparrow 1} b_{q^{z}}(t-l,-s+l+1)=\mathrm{B}(t-l,-s+l+1)$ for all $l(0 \leq l \leq r-1)$. Further, since the left hand side of (3.16) is equal to $\mathrm{B}(t-r+1,-s+r)=\Gamma(t-r+$ 1) $\Gamma(-s+r) / \Gamma(t-s+1)$, we have $t-s+1 \in \mathbf{Z}_{\leq 0}$, whence $t=\varphi(s)=s-v$ for some positive integer $v \in \mathbf{N}$ in the region $\operatorname{Re}(s)<1$. Since $\varphi(s)$ is meromorphic on $\mathbf{C}$, we have $\varphi(s)=s-v$ for all $s \in \mathbf{C}$. This proves the theorem.

\section{Remarks on $q$-analogues of $\zeta_{r}(s, z ; \omega)$.}

We introduce here a $q$-analogue of the Barnes multiple zeta function $\zeta_{r}(s, z ; \omega)$ for a general parameter $\omega:=\left(\omega_{1}, \ldots, \omega_{r}\right)$. Assume $\omega_{i}>0(1 \leq i \leq r)$ and $\operatorname{Re}(z)>0$. We define a $q$-analogue of $\zeta_{r}(s, z ; \omega)$ by the series

$$
\zeta_{q, r}(s, t, z ; \boldsymbol{\omega}):=\sum_{n_{1}, \ldots, n_{r} \geq 0} \frac{q^{n_{1} \omega_{1} t+n_{2} \omega_{2}(t-1)+\cdots+n_{r} \omega_{r}(t-r+1)}}{\left[n_{1} \omega_{1}+\cdots+n_{r} \omega_{r}+z\right]_{q}^{s}}
$$

We put $\zeta_{q, r}^{(v)}(s, z ; \boldsymbol{\omega}):=\zeta_{q, r}(s, s-v, z ; \boldsymbol{\omega})$ for $v \in \mathbf{N}$. The series $\zeta_{q, r}(s, t, z ; \boldsymbol{\omega})$ converges absolutely for $\operatorname{Re}(t)>r-1$. It is clear that $\zeta_{q, r}(s, t, z)=\zeta_{q, r}\left(s, t, z ; \mathbf{1}_{r}\right)$ where $\mathbf{1}_{r}:=$ $(\underbrace{1,1, \ldots, 1}_{r})$. By the following proposition, $\zeta_{q, r}(s, t, z ; \boldsymbol{\omega})$ is continued meromorphically to the whole $s, t$-plane. The proof can be obtained by the similar way to [5, Proposition 1] and [6, Proposition 2.9].

PROPOSITION 4.1. (i) The function $\zeta_{q, r}(s, t, z ; \omega)$ can be written as

$$
\zeta_{q, r}(s, t, z ; \boldsymbol{\omega})=(1-q)^{s} \sum_{l=0}^{\infty}\left(\begin{array}{c}
s+l-1 \\
l
\end{array}\right) q^{l z} \prod_{j=1}^{r}\left(1-q^{\omega_{j}(t-j+1+l)}\right)^{-1}
$$

This gives a meromorphic continuation of $\zeta_{q, r}(s, t, z ; \omega)$ to the whole $s, t$-plane with simple poles at $t \in j-1+\mathbf{Z}_{\leq 0}+\delta_{j} \mathbf{Z}(1 \leq j \leq r)$. Here $\delta_{j}:=2 \pi \sqrt{-1} /\left(\omega_{j} \log q\right)$.

(ii) The function $\zeta_{q, r}^{(v)}(s, z ; \omega)$ can be written as

$$
\zeta_{q, r}^{(v)}(s, z ; \omega)=(1-q)^{s} \sum_{l=0}^{\infty}\left(\begin{array}{c}
s+l-1 \\
l
\end{array}\right) q^{l z} \prod_{j=1}^{r}\left(1-q^{\omega_{j}(s-v-j+1+l)}\right)^{-1} .
$$


This gives a meromorphic continuation of $\zeta_{q, r}^{(v)}(s, z ; \boldsymbol{\omega})$ to the whole plane $\mathbf{C}$ with simple poles at the points in

$$
\begin{cases}j+\delta_{i} \mathbf{Z} \backslash\{0\} & \left(j \in \mathbf{Z}_{\leq 0}, 1 \leq i \leq r\right) \\ j+\delta_{i} \mathbf{Z} & (1 \leq j \leq v, 1 \leq i \leq r), \\ v+j+\delta_{i} \mathbf{Z} & (1 \leq j \leq r-1, j+1 \leq i \leq r)\end{cases}
$$

In particular, the poles of $\zeta_{q, r}^{(v)}(s, z ; \omega)$ on the real axis are given by $s=1,2, \ldots, r, r+$ $1, \ldots, r+v-1$.

(iii) Let $m \in \mathbf{Z}_{\geq 0}$. Then we have

$$
\begin{aligned}
\zeta_{q, r}^{(\nu)}(-m, z ; \boldsymbol{\omega})= & (1-q)^{-m}\left\{\sum_{l=0}^{m}(-1)^{l}\left(\begin{array}{c}
m \\
l
\end{array}\right) q^{l z} \prod_{j=1}^{r}\left(1-q^{\omega_{j}(-m-v+l-j+1)}\right)^{-1}\right. \\
& \left.+\frac{q^{(m+v-1) z}}{\log q} \sum_{l=1}^{r} \frac{(-1)^{m+1} m !(l+v-2) ! q^{l z}}{(l+m+v-1) ! \omega_{l}} \prod_{\substack{j=1 \\
j \neq l}}^{r}\left(1-q^{\omega_{j}(l-j)}\right)^{-1}\right\} .
\end{aligned}
$$

Proof. The formula (4.1) is obtained by the binomial theorem, whence (4.2) immediately follows. The formula (4.3) is derived from the fact $(s+m) /\left(1-q^{\omega_{l}(s+m)}\right)=$ $-1 /\left(\omega_{l} \log q\right)+O(s+m)$ as $s \rightarrow-m$.

These facts motivate the

CONJECTURE 4.2. Let $t=\varphi(s)$ be a meromorphic function on $\mathbf{C}$. Then the formula

$$
\lim _{q \uparrow 1} \zeta_{q, r}(s, \varphi(s), z ; \boldsymbol{\omega})=\zeta_{r}(s, z ; \boldsymbol{\omega}) \quad(s \in \mathbf{C})
$$

holds if and only if the function $\varphi(s)$ can be written as $\varphi(s)=s-v$ for some $\nu \in \mathbf{N}$.

In fact, since $\zeta_{1}(s, z ; \omega)=\omega^{-s} \zeta(s, z / \omega)$ and $\zeta_{q, 1}(s, t, z ; \omega)=[\omega]_{q}{ }^{-s} \zeta_{q^{\omega}}(s, t, z / \omega)$ for $\omega>0$, Conjecture 4.2 is true for $r=1$ by (3.4) and (3.11).

\section{A. An associated $q$-analogue of the gamma function}

In this appendix, we introduce a $q$-analogue of the gamma function defined via the $q$ analogue of the Hurwitz zeta function:

$$
\tilde{\zeta}_{q}(s, z):=\zeta_{q}^{(1)}(s, z) \times q^{z(s-1)}=\sum_{n=0}^{\infty} \frac{q^{(n+z)(s-1)}}{[n+z]_{q}^{s}} \quad(\operatorname{Re}(s)>1) .
$$

Note that by (3.1), we have

$$
\tilde{\zeta}_{q}(s, z)=\tilde{\zeta}_{q}(s, z+1)+\frac{q^{z(s-1)}}{[z]_{q}{ }^{s}} .
$$


Imitating the Lerch formula [8] (the zeta regularization)

$$
\left.\frac{\partial}{\partial s} \zeta(s, z)\right|_{s=0}=\log \frac{\Gamma(z)}{\sqrt{2 \pi}},
$$

we define a $q$-analogue $\tilde{\Gamma}_{q}(z)$ of the gamma function by

$$
\tilde{\Gamma}_{q}(z):=\exp \left(\left.\frac{\partial}{\partial s} \tilde{\zeta}_{q}(s, z)\right|_{s=0}-\left.\frac{\partial}{\partial s} \tilde{\zeta}_{q}(s, 1)\right|_{s=0}\right) .
$$

Then the function $\tilde{\Gamma}_{q}(z)$ is well-defined as a single valued meromorphic function. Indeed, let

$$
\tilde{\zeta}_{q}(s, z)=a_{0}(z ; q)+a_{1}(z ; q) s+a_{2}(z ; q) s^{2}+\cdots
$$

be the Taylor expansion of $\tilde{\zeta}_{q}(s, z)$ around $s=0$. Note that $\tilde{\zeta}_{q}(s, z)$ is holomorphic at $s=0$. Assume $\operatorname{Re}(z)>0$. Then, by Proposition 4.1, $\tilde{\zeta}_{q}(s, z)$ has the following expression;

$$
\tilde{\zeta}_{q}(s, z)=(1-q)^{s} \sum_{n=0}^{\infty}\left(\begin{array}{c}
s+n-1 \\
n
\end{array}\right) \frac{q^{z(s-1+n)}}{1-q^{s-1+n}} .
$$

Hence one can calculate the coefficient $a_{1}(z ; q)$ by the same manner performed in [7] as

$$
\begin{aligned}
a_{1}(z ; q)= & \sum_{n=2}^{\infty} \frac{1}{n} \frac{q^{(n-1) z}}{1-q^{n-1}}-z+\frac{1}{2}+\frac{1-z(1-q)}{(1-q)^{2}} q^{1-z} \log q \\
& -\left(\frac{q^{1-z}}{1-q}+\frac{1}{\log q}\right) \log (1-q) .
\end{aligned}
$$

Therefore $\tilde{\Gamma}_{q}(z)$ is meromorphic in the region $\operatorname{Re}(z)>0$. If $-1<\operatorname{Re}(z)<0$, by the ladder relation (A.1), we have

$$
a_{1}(z ; q)=q^{-z} \log q^{z}-q^{-z} \log \left(\frac{1-q^{z}}{1-q}\right)+a_{1}(z+1 ; q)
$$

Hence we have

$$
\tilde{\Gamma}_{q}(z)=\left(q^{-z}[z]_{q}\right)^{-q^{-z}} \tilde{\Gamma}_{q}(z+1)
$$

This gives a meromorphic continuation of $\tilde{\Gamma}_{q}(z)$ to the region $\operatorname{Re}(z)>-1$. Repeating the same procedure, we see that $\tilde{\Gamma}_{q}(z)$ can be extended as a meromorphic function on $\mathbf{C}$.

From Theorem 2.1 , by the Lerch formula, we have immediately

$$
\lim _{q \uparrow 1} \tilde{\Gamma}_{q}(z)=\Gamma(z) \quad\left(z \notin-\mathbf{Z}_{\geq 0}\right) .
$$

Moreover, $\tilde{\Gamma}_{q}(z)$ satisfies the following properties. 
Proposition A.1. We have

$$
\begin{aligned}
& \tilde{\Gamma}_{q}(z+1)=\left(q^{-z}[z]_{q}\right)^{q^{-z}} \tilde{\Gamma}_{q}(z), \\
& \tilde{\Gamma}_{q}(1)=1, \\
& \frac{d^{2}}{d z^{2}} \log \tilde{\Gamma}_{q}(z+1) \geq 0 \quad(z \geq 0) .
\end{aligned}
$$

In particular, for a positive integer $n$, we have

$$
\tilde{\Gamma}_{q}(n+1)=q^{-\sum_{k=1}^{n} k q^{-k}} \prod_{k=1}^{n}\left([k]_{q}\right)^{q^{-k}} .
$$

Proof. By the definition of $\tilde{\Gamma}_{q}(z),($ A.7) is obvious. The formula (A.6) is clear from (A.4). The assertion (A.9) follows from (A.6) and (A.7) by induction. To show the inequality (A.8), take the logarithm of $\tilde{\Gamma}_{q}(z)$ :

$$
\begin{aligned}
\log \tilde{\Gamma}_{q}(z)= & \sum_{n=2}^{\infty} \frac{1}{n} \frac{q^{z(n-1)}-q^{n-1}}{1-q^{n-1}}-z+1 \\
& +\frac{q^{-z}(1-(1-q) z)-1}{(1-q)^{2}} q \log q+\frac{1-q^{1-z}}{1-q} \log (1-q) .
\end{aligned}
$$

We calculate as

$$
\frac{d^{2}}{d z^{2}} \log \tilde{\Gamma}_{q}(z+1)=(\log q)^{2} \sum_{n=2}^{\infty} \frac{(n-1)^{2}}{n} \frac{q^{(z+1)(n-1)}}{1-q^{n-1}}+\frac{(\log q)^{2} q^{-z}}{(1-q)^{2}} \eta_{q}(z),
$$

where $\eta_{q}(z):=(\log q)(1-(1-q)(z+1))-(1-q) \log (1-q)+2(1-q)$. Therefore, it suffices to show that $\eta_{q}(z) \geq 0$ for all $0<q<1$ if $z \geq 0$, and this is indeed true. In fact, since $\frac{d}{d q} \eta_{q}(z) \leq 0$ for $0<q<1$, we conclude that $\eta_{q}(z) \geq \lim _{q \uparrow 1} \eta_{q}(z)=0$. Hence the proposition follows.

REMARK A.2. One can find the similar formulas to (A.6), (A.7) and (A.8) in the $q$ analogue of the Bohr-Morellup theorem for the Jackson $q$-gamma function in [2]. It has not yet been clarified that these properties characterize the function $\tilde{\Gamma}_{q}(z)$. $1:$

By the expression (A.2) again, $\tilde{\zeta}_{q}(s, z)$ has the following Laurent expansion around $s=$

$$
\tilde{\zeta}_{q}(s, z)=\frac{q-1}{\log q} \frac{1}{s-1}+\gamma_{q}(z)+O(s-1) \quad(\operatorname{Re}(z)>0),
$$

where

$$
\gamma_{q}(z):=\sum_{n=1}^{\infty} \frac{q^{n z}}{[n]_{q}}+(1-q)\left(-z+\frac{1}{2}-\frac{\log (1-q)}{\log q}\right) .
$$


We next show a $q$-analogue of the Lerch limit formula [8]:

$$
\lim _{s \rightarrow 1}\left(\zeta(s, z)-\frac{1}{s-1}\right)=-\frac{\Gamma^{\prime}}{\Gamma}(z) .
$$

Proposition A.3. It holds that

(A.14) $\gamma_{q}(z)=\lim _{s \rightarrow 1}\left(\tilde{\zeta}_{q}(s, z)-\frac{q-1}{\log q} \frac{1}{s-1}\right)=-\frac{q-1}{\log q} \frac{\tilde{\Gamma}_{q}^{\prime}}{\tilde{\Gamma}_{q}}(z)+C_{q}(z) \quad(\operatorname{Re}(z)>0)$,

where

$$
\begin{aligned}
C_{q}(z):= & \sum_{n=1}^{\infty} \frac{1}{n+1} \frac{q^{n z}}{[n]_{q}}+q^{1-z}+\frac{\log q}{1-q}(1-(1-q) z) q^{1-z}+\frac{1-q}{\log q} \\
& -q^{1-z} \log (1-q)-\frac{1-q}{\log q} \log (1-q)+\left(-z+\frac{1}{2}\right)(1-q)
\end{aligned}
$$

and $\lim _{q \uparrow 1} C_{q}(z)=0$. Put $\gamma_{q}:=\gamma_{q}(1)$. Then we have, in particular, $\lim _{q \uparrow 1} \gamma_{q}=\gamma$ where $\gamma=0.577215 \ldots$ denotes the Euler constant.

Proof. By (A.10), we have

$$
\begin{aligned}
\frac{\tilde{\Gamma}_{q}^{\prime}}{\tilde{\Gamma}_{q}}(z)= & \frac{\log q}{1-q}\left(\sum_{n=1}^{\infty} \frac{q^{z n}}{[n]_{q}}-\sum_{n=1}^{\infty} \frac{1}{n+1} \frac{q^{n z}}{[n]_{q}}\right)-1 \\
& -\frac{(1-q)+(1-(1-q) z) \log q}{(1-q)^{2}} q^{1-z} \log q+\frac{\log q}{1-q} q^{1-z} \log (1-q) .
\end{aligned}
$$

Plugging (A.12) into (A.15), we obtain the formula (A.14). It is straightforward to show the fact $\lim _{q \uparrow 1} C_{q}(z)=0$ when $\operatorname{Re}(z)>0$. Hence we have $\lim _{q \uparrow 1} \gamma_{q}=\gamma$ by the limit formulas (A.5), (A.13) and the facts $\Gamma(1)=1, \Gamma^{\prime}(1)=-\gamma$. This completes the proof.

Remark A.4. The $q$-analogue of the Lerch limit formula obtained in this paper is different from the one given in [7].

As a final remark, we give a $q$-analogue of the Gauss-Legendre formula.

Proposition A.5. Let $N \in \mathbf{N}$. Then we have

$$
\begin{aligned}
{[N]_{q}^{[1-N z]_{q}} \tilde{\Gamma}_{q^{N}}\left(\frac{1}{N}\right) \cdots \tilde{\Gamma}_{q^{N}}\left(\frac{N-1}{N}\right) \tilde{\Gamma}_{q}(N z) } \\
\quad=\tilde{\Gamma}_{q^{N}}(z) \tilde{\Gamma}_{q^{N}}\left(z+\frac{1}{N}\right) \cdots \tilde{\Gamma}_{q^{N}}\left(z+\frac{N-1}{N}\right) .
\end{aligned}
$$

Proof. The proof is straightforward from (A.10). 


\title{
References
}

[1] G. E. Andrews, R. Askey and R. Roy, Special Functions, Encyclopedia of math. and appl., vol. 71, Cambridge U.P. (1999).

[2] R. ASKEY, The $q$-gamma and $q$-Beta functions, Appl. Anal. 8 (1978), 125-141.

[3] E. BARnes, On the theory of the multiple gamma functions, Trans. Cambridge Philos. Soc. 19 (1904), 374425.

[ 4 ] K. Kimoto, N. Kurokawa, S. Matsumoto and M. Wakayama, Multiple finite Riemann zeta function, Acta Arith. 116 (2) (2005), 173-187.

[ 5 ] M. KANEKo, N. KUROKAWA and M. WAKAYAma, A variation of Euler's approach to values of the Riemann zeta function, Kyushu J. Math. 57 (2003), 175-192.

[6] K. Kawagoe, M. Wakayama and Y. Yamasaki, $q$-Analogues of the Riemann zeta, the Dirichlet $L$ functions, and a crystal zeta function, to appear in Forum. Math. (2006).

[ 7 ] N. Kurokawa and M. WaKayama, On $q$-analogue of the Euler constant and Lerch's limit formula, Proc. Amer. Math. Soc. 132 (2003), 935-943.

[ 8 ] M. LERCH., Dalši studie v oboru Malmsténovských řad, Rozpravy České Akad. 3 No. 28 (1894), 1-61.

[ 9 ] T. SEO, J. ChOI, J. GANG and B. OK, Note on the analytic continuation of the multiple Hurwitz zeta functions $\zeta_{n}(s, a)$, Kyungpook Math J. 33(2) (1993), 127-131.

[10] H. Tsumura, On modification of the $q$ - $L$-series and its applications, Nagoya Math. J. 164 (2001), 185-197.

[11] E. T. WhitTAKer and G. N. Watson, A Course of Modern Analysis 4th edn, Cambridge University Press (1927).

\author{
Present Address: \\ Graduate School of Mathematics, KyUshu University, \\ HAKOZAKI, FUKUOKA, 812-8581 JAPAN. \\ e-mail: yamasaki@math.kyushu-u.ac.jp
}

